\title{
The development of the method for identifying the hardening curves of materials based on the results of torsion tests of notched specimens
}

\author{
Mikhail Erpalov, ${ }^{1,}$, and Evgeny Panin ${ }^{2}$ \\ ${ }^{1}$ Ural Federal University, Metal Forming Department, 620002 Yekaterinburg 19 Mira Str., Russia \\ ${ }^{2}$ Karaganda Industrial University, Metal Forming Department, 101400 Temirtau 30 Republic Ave., \\ Kazakhstan
}

\begin{abstract}
The article is devoted to studying the rheological properties of metals and alloys in a cold state. The method is proposed to identify the hardening curve of a material based on torsion tests of round specimens with a notch. Determining the hardening curve is based on measuring the coordinates of points on the surface of the specimen after testing and determining the corresponding value of the torque. Based on these values the coefficients in an equation for the stress-strain dependence can be determined by minimizing the derived functional. The paper proposes the algorithm for the numerical implementation of the new identification method. The results of the computer simulation confirmed its effectiveness.
\end{abstract}

\section{Introduction}

The rheological properties of materials are the dependence of flow stress on the strain value, strain rate, temperature, and loading history [1]. This dependence is determined, as a rule, based on simple tests such as tension [2], compression [3, 4], torsion [5, 6], and some others. Torsion testing of specimens is relatively seldom used to study the rheological properties of the materials. At the same time, it is generally accepted that torsion is one of the most effective test methods, because during torsion, there is no effect of friction forces that appear on the contact surface with the tool, as occurs in compression, and there is also no viewed region of concentrated deformation, as in tensile tests.

When carrying out torsion tests using specimens with a cylindrical gauge length of radius $r$ and length $l$, it is assumed that the strain value corresponding to points on the specimen surface is distributed uniformly along the entire gauge length [5]:

$$
\varepsilon_{\text {surf }}=\frac{1}{\sqrt{3}} r \frac{\varphi}{l},
$$

where $\varphi$ is the twist angle of the specimen or the total angle of rotation of the test setup grips. But, in practice, the deformation is distributed non-uniformly [7].

\footnotetext{
* Corresponding author: m.v.erpalov@urfu.ru
} 
Even though during torsion there are no external signs that deformation is concentrated in some region, the inhomogeneity of deformation along the specimen can be determined experimentally. For this, along the generatrix of the specimen, before testing, it is necessary to apply a gauge mark, and after testing, measure the angle of this mark with the generatrix of the specimen $\gamma$ in a certain cross-section [8]. Knowing the angle $\gamma$, the strain value corresponding to the points on the specimen surface can be calculated by the formula:

$$
\varepsilon_{\text {surf }}=\frac{1}{\sqrt{3}} \operatorname{tg} \gamma=\frac{1}{\sqrt{3}} r \frac{d \varphi}{d z}
$$

where $z$ is the axial coordinate of the considered specimen cross-section.

The reason for the non-uniformity of the strain distribution can be the influence of nondeformable parts of the specimen, which restrain deformation in the transition zone to the gauge length, the low accuracy of the specimen dimensions, as well as the existence of hidden defects [9]. If the material shows softening effects, for example, in a hot state, the deformation non-uniformity increases. At the same time, the inhomogeneity of the strain distribution is stochastic and cannot be taken into account when processing experimental data, since it is not known how inhomogeneity changes during the test $[9,10]$.

The use of tubular specimens [11] instead of cylindrical ones, as well as carrying out tests on paired specimens, makes it possible to take into account the inhomogeneous distribution of deformation, as well as stresses only in the cross-section of the specimen. To take into account the inhomogeneous strain distribution in the longitudinal direction, the specimens with a round notch can be used [12]. In this case, it is impossible to ensure the constancy of the strain along the sample. However, it is possible to stabilize the position of the section with the maximum strain value.

To process the experimental data obtained using the notched specimens, it is necessary to establish the dependence of the strain in the minimum cross-section of the specimen on the twist angle $\varepsilon_{\text {surf }}(\varphi)$. However, as shown by the results of the earlier study [10], this function depends on the nature of the hardening curve of the material and the specific test conditions. In this case, the use of notched specimens requires a whole series of additional studies to establish these relationships. It significantly complicates the study of the rheological properties of materials and increases the cost of the research.

In this study, we propose a new approach to determine the hardening curves of the materials based on the results of testing the specimens with the notch for torsion in a cold state.

\section{The new method for identifying the hardening curves}

When testing the specimens for torsion the inhomogeneous distribution of stresses occurs in different cross-sections. This is because the material on the specimen axis does not deform during the entire test. In the absence of axial tensile or axial compressive stresses, it is assumed that in the direction from the center to the surface of the specimen, the strain increases from zero to a maximum value in proportion to the current radius:

$$
\varepsilon(\rho)=\varepsilon_{\text {surf }} \frac{\rho}{r} .
$$

When the material is in a cold state, there are no factors affecting the flow stresses other than the strain. Therefore, the stress distribution along the radius in the cross-section of the specimen repeats the material hardening curve: 


$$
\tau(\rho)=\tau\left(\varepsilon_{\text {surf }} \frac{\rho}{r}\right) .
$$

Knowing the stress distribution, the torque value can be calculated using the formula:

$$
M=2 \pi \int_{0}^{r} \tau\left(\varepsilon_{\text {surf }} \frac{\rho}{r}\right) \cdot \rho^{2} d \rho .
$$

In the case of testing the round specimens with the notch, an additional variable appears in Eq. 3 and 4, namely the longitudinal coordinate $z$ :

$$
\begin{gathered}
\varepsilon(\rho, z)=\varepsilon_{\text {surf }}(z) \cdot \frac{\rho}{r(z)}, \\
\tau(\rho, z)=\tau\left(\varepsilon_{\text {surf }}(z) \cdot \frac{\rho}{r(z)}\right) .
\end{gathered}
$$

However, the value of the torque is still constant and does not depend on the section choice:

$$
M(z)=2 \pi \int_{0}^{r(z)} \tau\left(\varepsilon_{\text {surf }}(z) \cdot \frac{\rho}{r(z)}\right) \cdot \rho^{2} d \rho=\text { const } .
$$

Eq. 6-8 correspond to a certain time instant of the test. Here, $r(z)$ is the function that describes the notch profile and $\varepsilon_{\text {surf }}(z)$ is the strain distribution on the notch surface along the specimen. The strain distribution, as well as the geometry of the specimen, can be determined experimentally on a basis of measuring the coordinates of the mark applied to the surface of the notch before testing along the generatrix.

If we set the material hardening law as a function of shear stress on the strain value, for example,

$$
\tau(\varepsilon)=A \cdot \varepsilon^{n}
$$

then it becomes possible to construct a functional of the form:

$$
J=\int_{z_{\min }}^{z_{\max }} 2 \pi \int_{0}^{r(z)} A\left(\varepsilon_{\text {surf }}(z) \cdot \frac{\rho}{r(z)}\right)^{n} \rho^{2} d \rho-M_{\text {test }} \mid d z,
$$

which contains as unknown variables only the coefficients of the hardening law. For determining the variables of the hardening law, it is necessary to minimize the functional $J$ :

$$
\left\{\begin{array}{l}
\frac{\partial J}{\partial A}=0 \\
\frac{\partial J}{\partial n}=0
\end{array}\right.
$$

In general, Eq. 10 can be written for any material hardening curve: 


$$
J=\int_{z_{\min }}^{z_{\max }}\left|M(z)-M_{\text {test }}\right| d z
$$

In Eq. $12, M_{\text {test }}$ is the current torque value determined experimentally, $z_{\min }$ and $z_{\max }$ are the minimum and maximum axial coordinates of the sections taken into account, for example, sections in which the strain value corresponding to the surface becomes zero.

Note that the minimum of the functional $J$ must correspond to the true hardening curve of the material even in the case of a nonlinear distribution of the strain along the radius. For this, Eq. 6 should be written taking into account additional experimental data on the actual strain distribution.

At any time instant of the test, there are points in the specimen that are not subject to shear deformation. They are located along the specimen axis. At the same time, on the surface of the minimum cross-section, the value of the strain is maximum. This means that the functional $J$ stores information on the entire hardening curve of the material in the range of strain values from zero to the maximum observed value. This also means that the hardening curve can be identified based on a single torque value corresponding to the distributions $r(z)$ and $\varepsilon_{\text {surf }}(z)$.

\section{The numerical implementation of the new method}

Eq. 12 allows solving the problem of identifying the material hardening curve if the dependences $r(z)$ and $\varepsilon_{\text {surf }}(z)$ are specified analytically. However, in real testing, any measurements are discrete. In this way, to use Eq. 12 the measurement results should be fitted to some analytical expressions. The choice of the approximation equations will determine the accuracy of the discrete data description. Taking into account the wide range of materials, shapes, and sizes of notches, it is more appropriate to use a numerical implementation of the method for identifying the material hardening curve.

Let us consider the case when the spatial coordinates of points $P_{i}\left(r_{i}, \varphi_{i}, z_{i}\right)$ corresponding to the gauge mark are determined at the end of the test. Then the strain distribution along the axis of the specimen can be calculated in the finite-difference form:

$$
\varepsilon_{\text {surf } i}=\frac{1}{\sqrt{3}} r_{i} \frac{\varphi_{i}-\varphi_{i-1}}{z_{i}-z_{i-1}} .
$$

It is proposed to perform numerical integration of shear stresses in the cross-section of the specimen by the trapezoidal method:

$$
M(i)=\frac{r_{i}}{n} \cdot\left[\pi \tau\left(\varepsilon_{\mathrm{surf} i}\right) \cdot r_{i}^{2}+2 \pi \sum_{i=1}^{n-1} \tau\left(\frac{i}{n} \varepsilon_{\mathrm{surf} i}\right) \cdot\left(\frac{i}{n} r_{i}\right)^{2}\right],
$$

where $n$ is the number of subdivisions of the integration domain from 0 to $r_{i}, \tau$ is the analytical expression for describing the hardening curve of the material accurate to unknown coefficients. The functional $J$ can be written in finite-difference form, taking into account the implementation of numerical integration by the trapezoidal method over the number of measurement points $k$ :

$$
J=\sum_{i=2}^{k} \frac{\left|M(i)-M_{\text {test }}\right|+\left|M(i-1)-M_{\text {test }}\right|}{2} \cdot\left(z_{i}-z_{i-1}\right) .
$$




\section{Evaluating the efficiency of the proposed method}

To evaluate the effectiveness of the proposed method for identifying the rheological properties of metals and alloys, it is necessary to perform a series of comparisons of the hardening curves determined using this method with the known hardening curves taken into account as true. The true hardening curves can be obtained from the results of testing specimens for tension, compression, etc., carried out in parallel with torsion tests. However, it cannot be guaranteed that no errors will be made while processing the experimental data obtained from the additional tests. Moreover, it cannot be guaranteed that the hypothesis of a single hardening curve is valid for current material and test conditions.

The most effective approach to test a new method for identifying the hardening curves is to use computer simulation based on the finite element method. This approach allows setting the material hardening curve and considering it as true. Computer simulation tools allow measuring the specimen, determining the fields of strain, stress, and calculating the values of the torque. If, after processing the data obtained from the computational experiment by the proposed method, the calculated hardening curve coincides or turns out to be very close to the specified one, then the method for identifying the hardening curves can be considered effective.

In this study, the Deform-2D software was used to solve the described problem. The specimen geometry, assigned arbitrarily, is shown in Fig. 1. The acuity of the notch, determined by the ratio of the radius of curvature $R$ to the minimum diameter $d$, is taken equal to 1 .

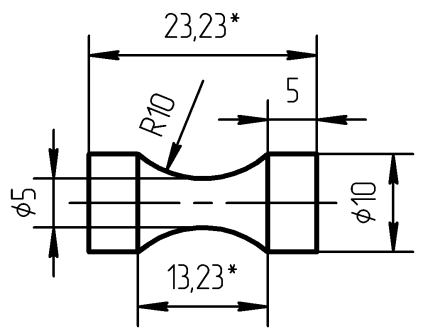

Fig. 1. The dimensions of the specimen used for the simulation.

The hardening curve of the material selected for the study is a power-law dependence of the flow stress on the strain value:

$$
\sigma_{\text {true }}=\sigma_{0}+A \cdot \varepsilon^{n}=200+200 \cdot \varepsilon^{0.5} .
$$

The specific values of the coefficients $\sigma_{0}, A$, and $n$ are chosen arbitrarily. The elastic properties of the material were not taken into account in this study. The simulation was carried out at the rate of twisting the specimen of $1 \mathrm{rpm}$ until the accumulation of the maximum strain value equal to 1 .

\section{The results of the study}

According to the results of the computational experiment, the axial and radial coordinates of points on the surface of the notch were determined corresponding to the end of the test (Fig. 2a). Determination of the angular coordinates is not necessary, since the Deform-2D software implements the ability to determine directly the strain values. In this way, Eq. 13 was not implemented for processing the results of the simulation. The distribution of the strain along the specimen is shown in Fig. $2 b$.

At the end of the test, the torque value was $M_{\text {test }}=6.99 \mathrm{~N} \cdot \mathrm{m}$. 


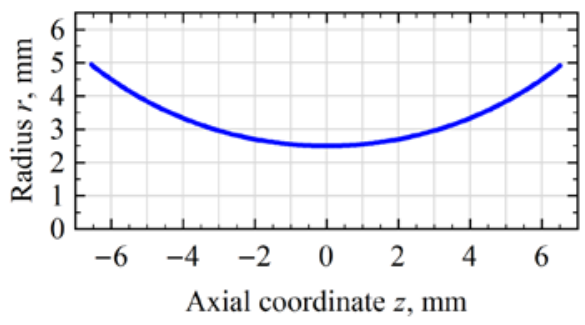

a

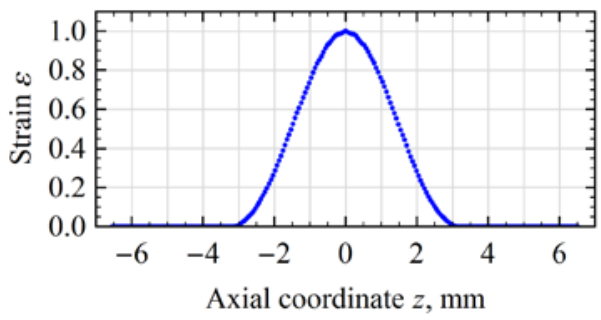

$\mathrm{b}$

Fig. 2. The specimen geometry after testing (a) and the strain distribution along the axis (b).

The results of the simulation were processed using the Wolfram Mathematica software. With its help, it was possible to implement the numerical algorithm for identifying the hardening curve of the material. While processing the experimental data it was taken into account that shear stresses should be replaced by normal ones. In addition, the linear distribution of the strain along the radius of the specimen was considered.

Note that the problem of minimizing the functional $J$ can be solved using any available optimization algorithm, for example, the gradient descent method, coordinate descent method, simplex method, etc. However, in this study, we used the direct search method, which, despite the large time costs, made it possible to study the functional $J$ values in a wide range of variation of the coefficients $\left(\sigma_{0}, A, n\right)$ in the vicinity of the known optimal point $(200,200,0.5)$.

For the convenience of presenting the results obtained, the problem of minimizing the functional $J$ was replaced by the problem of maximizing the parameter $\delta$ :

$$
\delta=\frac{M_{\text {test }}-J}{M_{\text {test }}} \cdot 100 \% .
$$

The objective function $\delta$ can take a maximum value of $100 \%$ only if in each crosssection of the specimen the calculated value of the torque is equal to the actual one.

The search for the extremum of the parameter $\delta$ and the corresponding values of the variables $\left(\sigma_{0}, A, n\right)$ was divided into two stages. At the first stage, with a large step of change in the values of the hardening law coefficients, the region was found in which the calculated extremum point is located. In Fig. 3a, this region corresponds to the value of the objective function $\delta=99.67 \%$. The calculation results show that the defined optimal region includes the true extremum point.

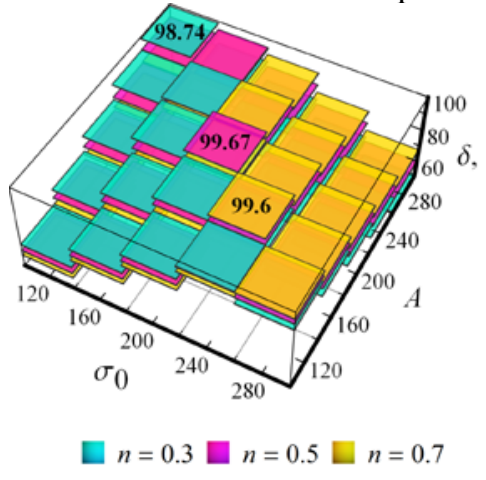

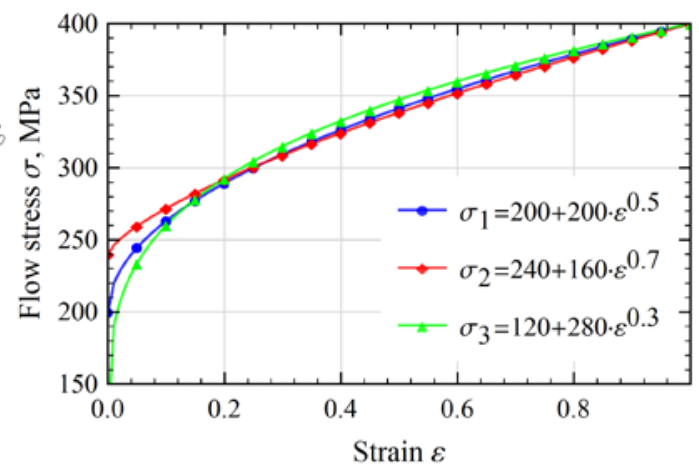

$\mathrm{b}$

Fig. 3. The results of calculating the objective function $\delta$ (a) and the defined optimal stress-strain curves (b). 
Despite the lying of the true extremum point in the region of the calculated one, it should be paid attention to two regions of the variables $\left(\sigma_{0}, A, n\right)$ with the center coordinates of $(120,280,0.3)$ and $(240,160,0.7)$. In these regions, there are no extremum points, but their corresponding values of the objective function $\delta$ equaled to 98.74 and $99.6 \%$ respectively differ from the calculated maximum $99.67 \%$ insignificantly. To understand the reason for such a high value of the objective function, we considered the corresponding hardening curves (Fig. 3b). As we can see from Fig. 3b, the second and third hardening curves corresponding to the peaks of the objective function are close to the first one corresponding to the calculated maximum of $\delta$. This means that all three hardening curves determine the torque in different sections of the specimen during deformation in a similar way. The only exception is the range of small values of the strain.

At the second stage of searching the extremum, due to a decrease in the step of variation, a more accurate value of the variables $\left(\sigma_{0}, A, n\right)$ corresponding to the maximum of the objective function $\delta$ was established. The results of optimization are shown in Fig. 4a. In Fig. 4b, in order of decreasing accuracy, three calculated hardening curves of the material are shown in comparison with the true hardening curve.

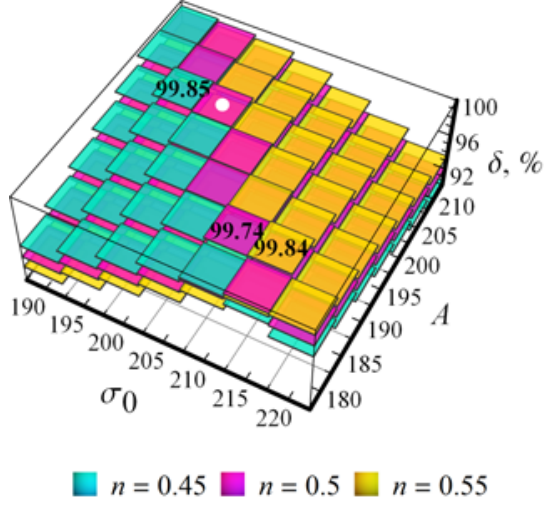

a

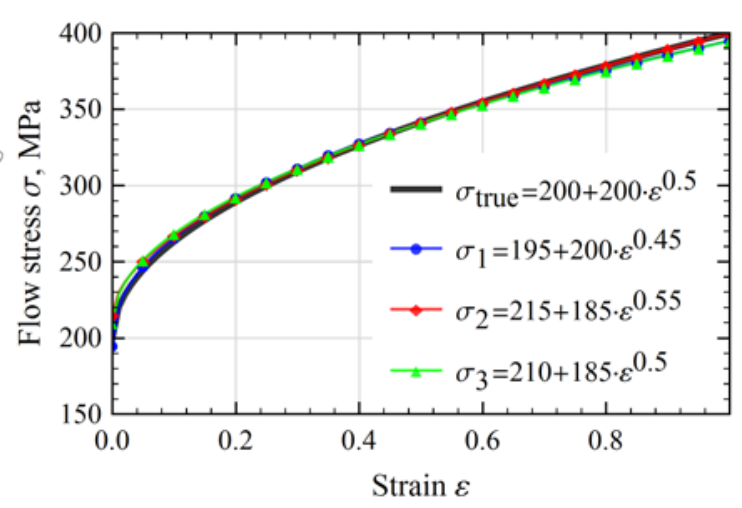

b

Fig. 4. The results of calculating the objective function $\delta$ (a) and the defined optimal stress-strain curves (b).

The results indicate that the calculated and true extremum points did not coincide. The true extremum point is marked with a white circle in Fig. 4a, and the calculated extremum has the value of the objective function equal to $99.85 \%$. The calculated extremum corresponds to the following hardening curve of the material:

$$
\sigma_{\text {opt }}=195+200 \cdot \varepsilon^{0.45} \text {. }
$$

Note that despite the deviation of the calculated optimal values of the hardening law coefficients from the true ones, the optimal hardening curve found with the use of the proposed identification method is practically impossible to distinguish from the specified one.

\section{Conclusions}

The results of the study show that the identification of the hardening curves based on the results of testing round specimens with a notch for torsion in a cold state is possible without additional studies aimed at obtaining the dependence of the strain in the minimum crosssection of the specimen on the twist angle. The proposed method for identifying hardening 
curves is based on the understanding of the strain and stress distributions over the volume of the specimen. Although the identification method does not guarantee the determination of the exact hardening law coefficients, it makes it possible to define the dependence of the flow stress values on the strain very accurately. The obtained data can be used directly in solving engineering or technological problems of metal forming, and can also represent starting values for a more accurate study of the material hardening curve using reverse computer simulation of the real test processes. The advantages of the proposed method include the ability to take into account the different kind of the strain distribution along the radius of the specimen, and the disadvantages include the need to assume one or another hardening law before identifying the unknown coefficients. This disadvantage can be overcome by setting the hardening curve in a discrete form and this approach has to be studied in further researches.

\section{References}

1. P. I. Polukhin, G. Ya. Gun, A. M. Galkin, Resistance to plastic deformation of metals and alloys (Metallurgiya, Moscow, 1976) (in Russian)

2. P. Bridgman, Studies in Large Plastic Flow and Fracture (Harvard University Press, Cambridge, Massachusetts, 1964)

3. A. I. Potapov, S. V. Gladkovsky, E. A. Kokovikhin, D. R. Salikhyanov, D. A. Dvoynikov, Diagnostics, Resource and Mechanics of materials and structures 2 24 (2015)

4. S. V. Smirnov, A. V. Konovalov, M. V. Myasnikova, Yu.V. Khalevitskiy, A.S. Smirnov, A.S. Igumnov, Phys. Mesomech. 21305 (2018)

5. A. Nadai, Theory of flow and fracture of solids (McGraw-Hill Book Company, New York, 1963)

6. D. S. Fields, W. A. Backofen, ASTM Proceeding 571259 (1957)

7. Yu. N. Loginov, S. L. Demakov, A. G. Illarionov, Russian Metallurgy (Metally) 2011 194 (2011)

8. A. A. Bogatov, O. I. Mizhiritsky, S. V. Smirnov, Resource of plasticity of metals in metal forming (Metallurgy, Moscow, 1984) (In Russian)

9. M. Erpalov, Solid State Phenom. 229323 (2020)

10. M.V. Erpalov, D. A. Pavlov, IOP Conf. Series: Materials Science and Engineering 971042025 (2020)

11. R.A. Vasin, A.A. Ilyushin, P.A. Mossakovsky, Mechanics of Solids 2177 (1994) (in Russian)

12. A. A. Bogatov, S. V. Smirnov, V.A. Krinitsin, et. al., Proceedings of Higher Educational Institutions. Ferrous Metallurgy 1237 (1981) (in Russian) 